\title{
LOCAL BEHAVIOUR OF SOLUTIONS OF STOCHASTIC INTEGRAL EQUATIONS
}

\author{
BY \\ WILLIAM J. ANDERSON
}

\begin{abstract}
Let $X$ denote the solution process of the stochastic equation $d X(t)$ $=a(X(t)) d t+\sigma(X(t)) d W(t)$. In this paper, conditions on $a(\cdot)$ and $\sigma(\cdot)$ are given under which the sample paths of $X$ are differentiable at $t=0$ with probability one. Variations of these results are obtained leading to a new uniqueness criterion for solutions of stochastic equations. If $\sigma(\cdot)$ is Hölder continuous with exponent greater than $\frac{1}{2}$ and $a(\cdot)$ satisfies a Lipschitz condition, it is shown that in the onedimensional case the above equation has only one continuous solution.
\end{abstract}

1. Introduction. Consider a one-dimensional stochastic integral equation

$$
X(t)=x_{0}+\int_{0}^{t} a(X(s)) d s+\int_{0}^{t} \sigma(X(s)) d W(s)
$$

where $x_{0} \in R . a(\cdot)$ and $\sigma(\cdot)$ are continuous real-valued functions on $R$, and $W$ is a one-dimensional Brownian motion process with $W(0)=0$. If $a(\cdot)$ and $\sigma(\cdot)$ satisfy Lipschitz conditions on $R$, the solution of (1) is unique and we can use uniqueness to show that the family of all such solutions as $x_{0}$ ranges over $R$ generates a continuous strong Markov process. If furthermore $\sigma\left(x_{0}\right)=0$, the strong Markov property may be used to show that

$$
\begin{aligned}
& \text { if } a\left(x_{0}\right)>0 \text {, then } \operatorname{Pr}\left(X(t) \geqq x_{0}, t \geqq 0\right)=1, \\
& \text { if } a\left(x_{0}\right)<0 \text {, then } \operatorname{Pr}\left(X(t) \leqq x_{0}, t \geqq 0\right)=1 .
\end{aligned}
$$

In the language of diffusions, $x_{0}$ is called in the first case a right shunt and in the second case a left shunt.

In this paper, we will reverse the steps of the above paragraph. Assuming that $\sigma(\cdot)$ satisfies on $R$ a Hölder condition with exponent greater than $\frac{1}{2}$, we will derive some results on the local behaviour of the solution $X$ of (1) similar to (2a) and (2b). This will be done in the multidimensional case. Then, assuming that $a(\cdot)$ satisfies a Lipschitz condition on $R$, we will use these results to prove that equation (1) can have only one continuous solution.

Background material on stochastic integrals and equations may be found in the books of K. Itô [3], H. P. McKean, Jr. [4], and A. V. Skorohod [5].

Received by the editors April 15, 1970.

AMS 1969 subject classifications. Primary 6075; Secondary 6062.

Key words and phrases. Stochastic integral equations, sample path behaviour, differentiability of solution, uniqueness of solution. 
This work was carried out at McGill University, Montreal, under a National Research Council of Canada Fellowship. The author would like to thank Professor D. A. Dawson for his generous guidance and assistance.

2. Notation and definitions. $(\Omega, \mathscr{F}, P)$ shall represent an arbitrary probability space on which is defined an $n$-dimensional Brownian motion process $W$ with $W(0)=0$ a.e. When $n \geqq 1$, we shall denote the independent component processes of $W$ by $W^{k}, k=1, \ldots, n$. $\left(\mathscr{F}_{t}, t \geqq 0\right)$ is an increasing family of sigma-subalgebras of $\mathscr{F}$ such that $W(t)$ is $\mathscr{F}_{t}$-measurable and such that $W(t+h)-W(t)$ and $\mathscr{F}_{t}$ are independent for any $t \geqq 0, h>0$.

A Markov time is a function $m: \Omega \rightarrow[0, \infty]$ such that $(m<t) \in \mathscr{F}_{t}$ for all $t \geqq 0$. We denote by $\mathscr{F}_{m}$ the sigma-algebra of events prior to $m$. $\mathscr{F}_{m}$ consists of all those $A \in \mathscr{F}$ such that $A \cap(m<t) \in \mathscr{F}_{t}$ for all $t \geqq 0$. If $m$ is a Markov time, the process $W^{+}$defined by $W^{+}(t)=W(t+m)-W(t), t \geqq 0$, is with respect to the measure $P(\cdot / m<+\infty)$ a new Brownian motion independent of $\mathscr{F}_{m}$.

A function $f:[0, \infty) \times \Omega \rightarrow R$ will be called nonanticipating if

(a) $f$ is measurable in the pair $(t, \omega)$,

(b) $f(t, \cdot)$ is $\mathscr{F}_{t}$-measurable for almost every $t \geqq 0$.

We shall also add the condition that

$$
P\left(\int_{0}^{t}|f(s, \cdot)|^{2} d s<+\infty, t \geqq 0\right)=1
$$

although this is not usually part of the definition. We will further say that $f$ is continuous if for almost every $\omega \in \Omega$ the function $f(\cdot, \omega)$ is continuous in $t$.

A function $f:[0, \infty) \times \Omega \rightarrow R^{n}$ will be called nonanticipating if each component $f_{k}, k=1, \ldots, n$, is nonanticipating. If $R^{n} \otimes R^{n}$ denotes the family of all real $n \times n$ matrices, a function $f:[0, \infty) \times \Omega \rightarrow R^{n} \otimes R^{n}$ will be called nonanticipating if each component function $f_{i k}(\cdot)$ of $f(\cdot)=\left[f_{i k}(\cdot)\right], i, k=1, \ldots, n$, is nonanticipating.

If $f$ is nonanticipating and has values in either $R, R^{n}$ or $R^{n} \otimes R^{n}$, we denote by $\int_{0}^{t} f(s) d W(s)$ the stochastic integral of $f$ over the interval $[0, t)$. If $f$ has values in $R$, $\int_{0}^{t} f(s) d W(s)$ is simply the one-dimensional Itô stochastic integral. If $f$ has values in $R^{n}, \int_{0}^{t} f(s) d W(s)$ is defined to be $\sum_{k=1}^{n} \int_{0}^{t} f_{k}(s) d W^{k}(s)$. If $f$ has values in $R^{n} \otimes R^{n}$, then $\int_{0}^{t} f(s) d W(s)$ is the $R^{n}$-valued random variable whose $i$ th component is given by $\sum_{k=1}^{n} \int_{0}^{t} f_{i k}(s) d W^{k}(s)$.

Finally we make the following remark. If $f$ is nonanticipating and has values in $R^{n}$, we may write

$$
\int_{0}^{t} f(s) d W(s)=e(j(t)), \quad t \geqq 0,
$$

where $e$ is a new one-dimensional Brownian motion process with $e(0)=0$ a.e. and $j$ is the function of $(t, \omega)$ defined for each $\omega$ by $j(t)=\int_{0}^{t}\|f(s)\|^{2} d s, t \geqq 0$. Here $\|\cdot\|$ denotes the Euclidean norm in $R^{n}$. We shall refer to the above as the random time substitution property. A proof may be found in McKean's book [4]. 
3. The law of the iterated logarithm for stochastic integrals. One can use the random time substitution property to obtain a law of the iterated logarithm for stochastic integrals. We give a proof below which depends on the following simple lemma: if $h:[0, \infty) \rightarrow[0, \infty)$ is such that $h(0)=0$ and the derivative $h^{\prime}(t)$ is continuous and nonnegative, then

$$
\lim _{t \rightarrow 0} \frac{h(t) \log \log 1 / h(t)}{t \log \log 1 / t}=h^{\prime}(0) .
$$

Alternate proofs of the following theorem may be found in [1] and [4].

THEOREM 3.1. Let $f:[0, \infty) \times \Omega \rightarrow R^{n}$ be a continuous nonanticipating function and put $Z(t)=\int_{0}^{t} f(s) d W(s), t \geqq 0$. Then

$$
\limsup _{t \rightarrow 0} \frac{Z(t)}{(2 t \log \log 1 / t)^{1 / 2}}=\|f(0)\| \quad \text { a.e. }
$$

Proof. Define $j(t)=\int_{0}^{t}\|f(s)\|^{2} d s, t \geqq 0$. According to the random time substitution property, $\int_{0}^{t} f(s) d W(s)=e(j(t)), t \geqq 0$, for some new Brownian motion $e$. Notice also that

$$
\lim _{t \rightarrow 0} \frac{j(t) \log \log 1 / j(t)}{t \log \log 1 / t}=j^{\prime}(0)=\|f(0)\|^{2} \quad \text { a.e. }
$$

Therefore

$$
\begin{aligned}
\limsup _{t \rightarrow 0} \frac{Z(t)}{(2 t \log \log 1 / t)^{1 / 2}} \\
\quad=\limsup _{t \rightarrow 0} \frac{e(j(t))}{(2 j(t) \log \log 1 / j(t))^{1 / 2}} \cdot \frac{(2 j(t) \log \log 1 / j(t))^{1 / 2}}{(2 t \log \log 1 / t)^{1 / 2}} \\
\quad=\limsup _{t \rightarrow 0} \frac{e(j(t))}{(2 j(t) \log \log 1 / j(t))^{1 / 2}} \cdot \lim \frac{(2 j(t) \log \log 1 / j(t))^{1 / 2}}{(2 t \log \log 1 / t)^{1 / 2}} \\
=\|f(0)\| \quad \text { a.e. }
\end{aligned}
$$

In the last step we have used the law of the iterated logarithm for the Brownian motion e. Q.E.D.

4. Sample path behaviour for solutions of stochastic equations. In this section, $a: R^{n} \rightarrow R^{n}$ and $\sigma: R^{n} \rightarrow R^{n} \otimes R^{n}$ are continuous functions. This means that each component $a_{k}(\cdot)$ of $a(\cdot)$ and $\sigma_{i k}(\cdot)$ of $\sigma(\cdot)=\left[\sigma_{i k}(\cdot)\right], i, k=1, \ldots, n$, is continuous. We shall assume that $X$ is a continuous nonanticipating function with values in $R^{n}$ such that the stochastic equation

$$
X(t)=X(0)+\int_{0}^{t} a(X(s)) d s+\int_{0}^{t} \sigma(X(s)) d W(s), \quad t \geqq 0,
$$


is satisfied. Here, $X(0)$ is an $\mathscr{F}_{0}$-measurable random variable with values in $R^{n}$. Equation (3) is equivalent to the system of $n$ equations

$$
\begin{aligned}
X_{i}(t)= & X_{i}(0)+\int_{0}^{t} a_{i}(X(s)) d s \\
& +\sum_{k=1}^{n} \int_{0}^{t} \sigma_{i k}(X(s)) d W^{k}(s), \quad t \geqq 0,
\end{aligned}
$$

$i=1, \ldots, n$. Let us define the function $b: R^{n} \rightarrow R^{n} \otimes R^{n}$ by $b(\cdot)=\sigma(\cdot) \sigma^{T}(\cdot)$, where $\sigma^{T}(\cdot)$ is the transpose of the matrix $\sigma(\cdot)$. Then we have the following law of the iterated logarithm for the function $X$.

\section{THEOREM 4.1.}

$$
\begin{aligned}
\limsup _{t \rightarrow 0} \frac{\left(X_{i}(t)+X_{j}(t)\right)-\left(X_{i}(0)+X_{j}(0)\right)}{(2 t \log \log 1 / t)^{1 / 2}} & \\
=\left(\left(b_{i i}(X(0))\right)^{1 / 2}+2 b_{i j}(X(0))+b_{j j}(X(0))\right) \text { a.e. } &
\end{aligned}
$$

for any $i, j=1, \ldots, n$.

Proof. Using (4), we have

$$
\begin{aligned}
& \frac{\left(X_{i}(t)+X_{j}(t)\right)-\left(X_{i}(0)+X_{j}(0)\right)}{(2 t \log \log 1 / t)^{1 / 2}} \\
& =\frac{\int_{0}^{t}\left(a_{i}(X(s))+a_{j}(X(s))\right) d s}{(2 t \log \log 1 / t)^{1 / 2}}+\sum_{k=1}^{n} \frac{\int_{0}^{t}\left(\sigma_{i k}(X(s))+\sigma_{j k}(X(s))\right) d W^{k}(s)}{(2 t \log \log 1 / t)^{1 / 2}} .
\end{aligned}
$$

Now $\int_{0}^{t}\left(a_{i}(X(s))+a_{j}(X(s))\right) d s$ is continuously differentiable in $t$ and therefore

$$
\lim _{t \rightarrow 0} \frac{\int_{0}^{t}\left(a_{i}(X(s))+a_{j}(X(s))\right) d s}{(2 t \log \log 1 / t)^{1 / 2}}=0 \quad \text { a.e. }
$$

Thus, taking lim sup $\operatorname{su}_{t \rightarrow 0}$ of both sides of the above equation, we have according to Theorem 3.1,

$$
\begin{aligned}
& \limsup _{t \rightarrow 0} \frac{\left(X_{i}(t)+X_{j}(t)\right)-\left(X_{i}(0)+X_{j}(0)\right)}{(2 t \log \log 1 / t)^{1 / 2}} \\
& =\left[\sum_{k=1}^{n}\left(\sigma_{i k}(X(0))+\sigma_{j k}(X(0))\right)^{2}\right]^{1 / 2} \\
& =\left(b_{i i}(X(0))+2 b_{i j}(X(0))+b_{j j}(X(0))\right)^{1 / 2} \text { a.e. Q.E.D. }
\end{aligned}
$$

Consider now the case where $X(0) \equiv z \in R^{n}$. By the above theorem,

$$
\limsup _{t \rightarrow 0} \frac{\left(X_{i}(t)+X_{j}(t)\right)-\left(z_{i}+z_{j}\right)}{(2 t \log \log 1 / t)^{1 / 2}}=\left(b_{i i}(z)+2 b_{i j}(z)+b_{j j}(z)\right)^{1 / 2}
$$

almost everywhere for $i, j=1, \ldots, n$. In this way the solution $X$ of equation (3) with initial condition $X(0) \equiv z$ determines the matrix $b(z)$. For example, if we set 
$i=j$, we can obtain the diagonal elements $b_{i i}(z), i=1, \ldots, n$, from the equation

$$
\limsup _{t \rightarrow 0} \frac{X_{i}(t)-z_{i}}{(2 t \log \log 1 / t)^{1 / 2}}=\left(b_{i i}(z)\right)^{1 / 2} \quad \text { a.e. }
$$

and once these are known, we can calculate the off-diagonal elements $b_{i j}(z)$ from (5). A natural question is whether the solution $X$ determines the vector $a(z)$ in a similar way. We shall see in Theorem 4.2 that under additional conditions on the function $b(\cdot)$, the answer to this question is yes.

If $b_{i i}(z)=0$, then from (6) we have

$$
\limsup _{t \rightarrow 0} \frac{X_{i}(t)-z_{i}}{(2 t \log \log 1 / t)^{1 / 2}}=0 \quad \text { a.e. }
$$

Since one can also show that

$$
\liminf _{t \rightarrow 0} \frac{X_{i}(t)-z_{i}}{(2 t \log \log 1 / t)^{1 / 2}}=-\left(b_{i i}(z)\right)^{1 / 2}=0 \quad \text { a.e., }
$$

we can conclude that

$$
\lim _{t \rightarrow 0} \frac{X_{i}(t)-z_{i}}{(2 t \log \log 1 / t)^{1 / 2}}=0 \quad \text { a.e. }
$$

The question is whether we can strengthen this result. In other words, is there a function $h(t)$ such that $\lim _{t \rightarrow 0}\left(X_{i}(t)-z_{i}\right) / h(t)$ exists and is nonzero?

The following theorem answers the above two questions simultaneously.

THEOREM 4.2. Assume that $X(0) \equiv z \in R^{n}$ and fix $i$ between 1 and $n$. Suppose that the function $b_{i i}(\cdot)$ satisfies the following conditions:

(i) $b_{i i}(z)=0$,

(ii) there is $K \geqq 0, \delta>0$, and $\alpha>0$ such that $b_{i i}(x) \leqq K\left|x_{i}-z_{i}\right|^{2 \alpha}$ whenever $\left|x_{i}-z_{i}\right|$ $<\delta$.

Then

(1) $\lim _{t \rightarrow 0}\left(X_{i}(t)-z_{i}\right) / t^{\lambda}=0$ a.e. if $\lambda<\min (1, \beta)$,

(2) $\lim _{t \rightarrow 0}\left(X_{i}(t)-z_{i}\right) / t=a_{i}(z)$ a.e. if $\beta>1$,

(3) $\lim _{t \rightarrow 0}\left(X_{i}(t)-z_{i}\right) / t^{\lambda}=0$ a.e. if $a_{i}(\cdot)=0$ and $\lambda<\beta$, where

$$
\begin{aligned}
\beta=(1 / 2) \sum_{p=0}^{\infty} \alpha^{p} & =+\infty & & \text { if } \alpha \geqq 1, \\
& =1 / 2(1-\alpha) & & \text { if } 0<\alpha<1 .
\end{aligned}
$$

Proof. For every $\lambda \in R$, we have

$$
\lim _{t \rightarrow 0} \frac{X_{i}(t)-z_{i}}{t^{\lambda}}=\lim _{t \rightarrow 0} \frac{\int_{0}^{t} a_{i}(X(s)) d s}{t^{\lambda}}+\lim _{t \rightarrow 0} \frac{\sum_{k=1}^{n} \int_{0}^{t} \sigma_{i k}(X(s)) d W^{k}(s)}{t^{\lambda}}
$$

provided the limits on the right-hand side exist. Now

$$
\begin{aligned}
\lim _{t \rightarrow 0} \frac{1}{t^{\lambda}} \int_{0}^{t} a_{i}(X(s)) d s & =0 & & \text { a.e. if } \lambda<1, \\
& =a_{i}(z) & & \text { a.e. if } \lambda=1, \\
& =0 & & \text { a.e. if } a_{i}(\cdot)=0,
\end{aligned}
$$


so that the results of the theorem are mainly dependent on $\lambda$ and $a_{i}(\cdot)$. As will become apparent from the following proof, it suffices to prove part (3), where we assume $a_{i}(\cdot)=0$.

Hence assume $a_{i}(\cdot)=0$. We are then concerned with the stochastic equation

$$
X_{i}(t)-z_{i}=\sum_{k=1}^{n} \int_{0}^{t} \sigma_{i k}(X(s)) d W^{k}(s), \quad t \geqq 0 .
$$

Let us show by induction that

$$
\lim _{t \rightarrow 0}\left(X_{i}(t)-z_{i}\right) / t^{\lambda}=0 \quad \text { a.e. if } \lambda<\left(1+\alpha+\alpha^{2}+\cdots+\alpha^{m}\right) / 2
$$

for some integer $m \geqq 0$. Because $\lim _{t \rightarrow 0}\left(X_{i}(t)-z_{i}\right) /(2 t \log \log 1 / t)^{1 / 2}=0$ a.e., it is an easy matter to show that our statement is true for $m=0$. Hence let us fix $m \geqq 0$ and assume that $\lim _{t \rightarrow 0}\left(X_{i}(t)-z_{i}\right) / t^{\lambda}=0$ a.e. for any $\lambda<\left(1+\alpha+\cdots+\alpha^{m}\right) / 2$. Suppose that $0<\lambda^{\prime}<\left(1+\alpha+\cdots+\alpha^{m+1}\right) / 2$. Then $-1 / 2 \alpha<\left(\lambda^{\prime}-\frac{1}{2}\right) / \alpha<\left(1+\alpha+\cdots+\alpha^{m}\right) / 2$. Now choose $\lambda$ such that $\left(\lambda^{\prime}-\frac{1}{2}\right) / \alpha<\lambda<\left(1+\alpha+\cdots+\alpha^{m}\right) / 2$ and note that $2 \alpha \lambda+1$ $>2 \lambda^{\prime}>0$. Making use of the random time substitution property, we can write $X_{i}(t)-z_{i}=e(j(t)), t \geqq 0$, where $e$ is a new Brownian motion process and $j(t)$ $=\int_{0}^{t} b_{i i}(X(s)) d s$. According to the induction hypothesis there is $\delta^{\prime}>0$ such that if $0<t \leqq \delta^{\prime}$, then $\left(X_{i}(t)-z_{i}\right)^{2} / t^{2 \lambda} \leqq 1$ and therefore $\left(X_{i}(t)-z_{i}\right)^{2} \leqq t^{2 \lambda} \leqq\left(\delta^{\prime}\right)^{2 \lambda}$. If we assume as we may that $\left(\delta^{\prime}\right)^{\lambda}<\delta$, then

$$
\begin{aligned}
0 & \leqq j(t)=\int_{0}^{t} b_{i i}(X(s)) d s \leqq K \int_{0}^{t}\left|X_{i}(s)-z_{i}\right|^{2 \alpha} d s \\
& \leqq K \int_{0}^{t} s^{2 \alpha \lambda} d s=\frac{K t^{2 \alpha \lambda+1}}{2 \alpha \lambda+1}
\end{aligned}
$$

for all $t \in\left[0, \delta^{\prime}\right]$. Putting $K^{\prime}=K /(2 \alpha \lambda+1)$, we get

$$
\begin{aligned}
0 & \leqq \lim _{t \rightarrow 0} \frac{j(t) \log \log 1 / j(t)}{t^{2 \lambda^{\prime}}} \leqq \lim _{t \rightarrow 0} \frac{K^{\prime} t^{2 \alpha \lambda+1} \log \log 1 / K^{\prime} t^{2 \alpha \lambda+1}}{t^{2 \lambda^{\prime}}} \\
& \leqq \lim _{t \rightarrow 0} \frac{K^{\prime} t^{2 \alpha \lambda+1} \log 1 / K^{\prime} t^{2 \alpha \lambda+1}}{t^{2 \lambda^{\prime}}} \\
& \left.=\lim _{u \rightarrow+\infty} \frac{K^{\prime} \log \left(u / K^{\prime}\right)}{u^{\left(2 \alpha \lambda+1-2 \lambda^{\prime}\right) /(2 \alpha \lambda+1)}} \quad \text { (put } u=1 / t^{2 \alpha \lambda+1}\right) \\
& =0
\end{aligned}
$$

by L'Hôpital's rule because $\left(2 \alpha \lambda+1-2 \lambda^{\prime}\right) /(2 \alpha \lambda+1)>0$. Thus

so that

$$
\lim _{t \rightarrow 0} \frac{j(t) \log \log 1 / j(t)}{t^{2 \lambda^{\prime}}}=0
$$

$$
\begin{aligned}
\limsup _{t \rightarrow 0} \frac{X_{i}(t)-z_{i}}{t^{\lambda^{\prime}}} & =\limsup _{t \rightarrow 0} \frac{e(j(t))}{(2 j(t) \log \log 1 / j(t))^{1 / 2}} \cdot \frac{(2 j(t) \log \log 1 / j(t))^{1 / 2}}{t^{\lambda^{\prime}}} \\
& =\limsup _{t \rightarrow 0} \frac{e(j(t))}{(2 j(t) \log \log 1 / j(t))^{1 / 2}} \cdot \lim \frac{(2 j(t) \log \log 1 / j(t))^{1 / 2}}{t^{\lambda^{\prime}}} \\
& =0 \text { a.e. }
\end{aligned}
$$


Similarly we can show that $\lim _{i \rightarrow 0}\left(X_{i}(t)-z_{i}\right) / t^{\lambda^{\prime}}=0$ a.e. The induction is therefore complete and we have shown that $\lim _{t \rightarrow 0}\left(X_{i}(t)-z_{i}\right) / t^{\lambda}=0$ a.e. if $\lambda<\left(1+\alpha+\cdots+\alpha^{m}\right) / 2$ for some $m \geqq 0$.

The proof is finished if one notes that $\lambda<(1 / 2) \sum_{p=0}^{\infty} \alpha^{p}$ implies that $\lambda<\left(1+\alpha+\cdots+\alpha^{m}\right) / 2$ for some large enough integer $m$. Q.E.D.

COROLlaRY. In addition to the hypotheses of Theorem 4.2 assume that $\alpha>\frac{1}{2}$.

(1) If $a_{i}(z)>0$, then $P\left[X_{i}(t)>z_{i}\right.$ for all sufficiently small $\left.t>0\right]=1$.

(2) If $a_{i}(z)<0$, then $P\left[X_{i}(t)<z_{i}\right.$ for all sufficiently small $\left.t>0\right]=1$.

Proof. The assumption that $\alpha>\frac{1}{2}$ implies that $\beta>1$ and so by part (2) of Theorem 4.2,

$$
\lim _{t \rightarrow 0} \frac{X_{i}(t)-z_{i}}{t}=a_{i}(z) \text { a.e. }
$$

Results (1) and (2) of the corollary are easily deduced from this. Q.E.D.

If we make the extra assumption that the functions $\sigma_{i k}(\cdot), k=1, \ldots, n$, depend only on the $i$ th component $x_{i}$ of $x$ for each $x \in R^{n}$, we can strengthen the results of the above corollary to show that $z$ is a right shunt for $X_{i}$ if $a_{i}(z)>0$ and a left shunt if $a_{i}(z)<0$. The proof of this fact is similar to the proof of Theorem 5.2.

5. A comparison theorem for solutions of stochastic equations. In this section, $a: R^{n} \rightarrow R^{n}, c: R^{n} \rightarrow R^{n}$ and $\sigma: R^{n} \rightarrow R^{n} \otimes R^{n}$ are continuous functions. $X(0)$ is an $\mathscr{F}_{0}$-measurable random vector and $X$ and $Y$ are continuous nonanticipating functions satisfying

$$
\begin{array}{ll}
X(t)=X(0)+\int_{0}^{t} a(X(s)) d s+\int_{0}^{t} \sigma(X(s)) d W(s), & t \geqq 0, \\
Y(t)=X(0)+\int_{0}^{t} c(Y(s)) d s+\int_{0}^{t} \sigma(Y(s)) d W(s), & t \geqq 0 .
\end{array}
$$

THEOREM 5.1. For a fixed $i$ between 1 and $n$, suppose the functions $\sigma_{i k}(\cdot)$, $k=1, \ldots, n$, satisfy the condition

$$
\left|\sigma_{i k}(x)-\sigma_{i k}(y)\right| \leqq K\left|x_{i}-y_{i}\right|^{\alpha}, \quad x, y \in R^{n},
$$

where $K \geqq 0$ and $\alpha>\frac{1}{2}$.

Then

$$
\lim _{t \rightarrow 0} \frac{X_{i}(t)-Y_{i}(t)}{t}=a_{i}(X(0))-c_{i}(X(0)) \text { a.e. }
$$

Proof. Since

$$
X_{i}(t)-Y_{i}(t)=\int_{0}^{t}\left[a_{i}(X(s))-c_{i}(Y(s))\right] d s+\sum_{k=1}^{n} \int_{0}^{t}\left[\sigma_{i k}(X(s))-\sigma_{i k}(Y(s))\right] d W^{k}(s)
$$

we get

$$
\lim _{t \rightarrow 0} \frac{X_{i}(t)-Y_{i}(t)}{(2 t \log \log 1 / t)^{1 / 2}}=0 \quad \text { a.e. }
$$


after applying Theorem 4.1. We shall only sketch the rest of the proof, since it is similar to that of Theorem 4.2 part (2).

Let $N$ be a nonnegative integer such that

$$
\left(1+\alpha+\cdots+\alpha^{N}\right) / 2 \leqq 1<\left(1+\alpha+\cdots+\alpha^{N+1}\right) / 2 .
$$

We shall show that $\lim _{t \rightarrow 0}\left(X_{i}(t)-Y_{i}(t)\right) / t^{\lambda}=0$ a.e. if $\lambda<\left(1+\alpha+\cdots+\alpha^{N}\right) / 2$. We proceed by induction. Suppose for some fixed $m$ with $0 \leqq m<N$ that $\lim _{t \rightarrow 0}\left(X_{i}(t)-Y_{i}(t)\right) / t^{\lambda}=0$ a.e. if $\lambda<\left(1+\alpha+\cdots+\alpha^{m}\right) / 2$ (this is true for $m=0$ ). Choose $\lambda^{\prime}$ with $0<\lambda^{\prime}<\left(1+\alpha+\cdots+\alpha^{m+1}\right) / 2$. As in Theorem 4.2, we can choose $\lambda$ such that $\left(\lambda^{\prime}-\frac{1}{2}\right) / \alpha<\lambda<\left(1+\alpha+\cdots+\alpha^{m}\right) / 2$. Again we write

$$
X_{i}(t)-Y_{i}(t)=\int_{0}^{t}\left[a_{i}(X(s))-c_{i}(X(s))\right] d s+e(j(t)), \quad t \geqq 0,
$$

where $e$ is a new Brownian motion and

$$
j(t)=\int_{0}^{t} \sum_{k=1}^{n}\left[\sigma_{i k}(X(s))-\sigma_{i k}(Y(s))\right]^{2} d s .
$$

By the induction hypothesis there is $\delta>0$ such that if $t \in[0, \delta]$ then $\left(X_{i}(t)-Y_{i}(t)\right)^{2}$ $\leqq t^{2 \lambda}$. Using this, it is easy to show that $0 \leqq j(t) \leqq n K t^{2 \alpha \lambda+1} /(2 \alpha \lambda+1)$ if $t \in[0, \delta]$, and therefore that $\lim _{t \rightarrow 0}(j(t) \log \log 1 / j(t)) / t^{\lambda^{\prime}}=0$ a.e. Hence

$$
\begin{aligned}
\limsup _{t \rightarrow 0} \frac{X_{i}(t)-Y_{i}(t)}{t^{\lambda^{\prime}}}= & \lim _{t \rightarrow 0} \frac{\int_{0}^{t}\left[a_{i}(X(s))-c_{i}(Y(s))\right] d s}{t^{\lambda^{\prime}}} \\
& +\limsup _{t \rightarrow 0} \frac{e(j(t))}{(2 j(t) \log \log 1 / j(t))^{1 / 2}} \\
& \cdot \lim _{t \rightarrow 0} \frac{(2 j(t) \log \log 1 / j(t))^{1 / 2}}{t^{\lambda^{\prime}}} \\
= & 0 \quad \text { a.e. }
\end{aligned}
$$

Since also $\lim _{\inf _{t \rightarrow 0}}\left(X_{i}(t)-Y_{i}(t)\right) / t^{\lambda^{\prime}}=0$ a.e., this completes the induction.

We have shown that $\lim _{t \rightarrow 0}\left(X_{i}(t)-Y_{i}(t)\right) / t^{\lambda}=0$ a.e. if $\lambda<\left(1+\alpha+\cdots+\alpha^{N}\right) / 2$. Carrying the induction one step further shows that

$$
\lim _{t \rightarrow 0} \frac{\sum_{k=1}^{n} \int_{0}^{t}\left[\sigma_{i k}^{i}(X(s))-\sigma_{i k}(Y(s))\right] d W^{k}(s)}{t^{\lambda}}=0 \quad \text { a.e. }
$$

if $\lambda<\left(1+\alpha+\cdots+\alpha^{N+1}\right) / 2$, in particular when $\lambda=1$. Thus

$$
\begin{aligned}
\lim _{t \rightarrow 0} & \frac{X_{i}(t)-Y_{i}(t)}{t} \\
& =\lim _{t \rightarrow 0} \frac{\int_{0}^{t}\left[a_{i}(X(s))-c_{i}(Y(s))\right] d s}{t}+\lim _{t \rightarrow 0} \frac{\sum_{k=1}^{n} \int_{0}^{t}\left[\sigma_{i k}(X(s))-\sigma_{i k}(Y(s))\right] d W^{k}(s)}{t} \\
& =a_{i}(X(0))-c_{i}(X(0)) \text { a.e. Q.E.D. }
\end{aligned}
$$


THEOREM 5.2. In addition to the hypotheses of Theorem 5.1, assume that the functions $\sigma_{i k}(\cdot), k=1, \ldots, n$, depend only on the ith component $x_{i}$ of $x \in R^{n}$. Also suppose that $a_{i}(x)>c_{i}(x)$ for each $x \in R^{n}$. Then

$$
P\left[X_{i}(t) \geqq Y_{i}(t) \text { for all } t \geqq 0\right]=1 .
$$

Proof. According to the above theorem,

$$
\lim _{t \rightarrow 0} \frac{X_{i}(t)-Y_{i}(t)}{t}=a_{i}(X(0))-c_{i}(X(0))>0 \text { a.e. }
$$

Hence we certainly have

$$
P\left[X_{i}(t)>Y_{i}(t) \text { for all sufficiently small } t>0\right]=1 .
$$

Define the function $m: \Omega \rightarrow[0,+\infty]$ by

$$
\begin{aligned}
m(\omega) & =\inf \left[t>0 \mid X_{i}(t, \omega)-Y_{i}(t, \omega)<0\right], \\
& =+\infty \quad \text { if } X_{i}(t, \omega)-Y_{i}(t, \omega) \geqq 0 \text { for all } t \geqq 0 .
\end{aligned}
$$

$m$ is a Markov time, because if $t>0$, then

$$
(m \geqq t)=\bigcap_{r \text { rational; } r \in[0, t]}\left(X_{i}(r)-Y_{i}(r) \geqq 0\right) \in \mathscr{F}_{t} .
$$

Put $D=(m<+\infty)$ and assume that $P(D)>0$. Then we can define the probability measure $P^{\prime}(\cdot)=P(\cdot / D)$ on $\mathscr{F}$. We shall also define

$$
\begin{array}{rlrl}
W^{+}(t) & =W(t+m)-W(m), & t \geqq 0, \omega \in D, \\
X^{+}(t) & =X(t+m), & t \geqq 0, \omega \in D, \\
Y^{+}(t) & =Y(t+m), & t \geqq 0, \omega \in D, \\
\mathscr{F}_{t+m}= & \text { the sigma-algebra of events prior to } \\
& \text { the Markov time } t+m, \quad t \geqq 0 .
\end{array}
$$

Because of the continuity of $X$ and $Y$, we note that $P^{\prime}\left[X^{+}(0)=Y^{+}(0)\right]=1$.

It has already been mentioned that $W^{+}$is a Brownian motion process on $\left(\Omega, \mathscr{F}, P^{\prime}\right)$ independent of $\mathscr{F}_{m}$. The function $\sigma\left(X^{+}(t)\right)$ is nonanticipating with respect to the family $\left(\mathscr{F}_{t+m}, t \geqq 0\right)$ and we can define in the usual way the stochastic integrals $\int_{0}^{t} \sigma_{i k}\left(X^{+}(s)\right) d W^{+k}(s), t \geqq 0$, with respect to the space $\left(\Omega, \mathscr{F}, P^{\prime}\right)$, the Brownian motion $W^{+}$, and the family $\left(\mathscr{F}_{t+m}, t \geqq 0\right)$. Moreover it is not too difficult to show that

$$
\int_{0}^{t} \sigma_{i k}\left(X^{+}(s)\right) d W^{+k}(s)=\int_{m}^{t+m} \sigma_{i k}(X(s)) d W^{k}(s), \quad t \geqq 0,
$$

almost everywhere on $D$ with respect to $P$, or equivalently almost everywhere with respect to $P^{\prime}$. The same thing may of course be said for the integral $\int_{0}^{t} \sigma_{i k}\left(Y^{+}(s)\right) d W^{+k}(s)$. 
For any $t \geqq 0$, we can therefore write

$$
\begin{aligned}
X^{+}(t)-Y^{+}(t)= & X(t+m)-Y(t+m) \\
= & X(m)-Y(m)+\int_{t}^{t+m}\left[a_{i}(X(s))-c_{i}(Y(s))\right] d s \\
& +\sum_{k=1}^{n} \int_{t}^{t+m}\left[\sigma_{i k}(X(s))-\sigma_{i k}(Y(s))\right] d W^{k}(s) \\
= & \int_{0}^{t}\left[a_{i}\left(X^{+}(s)\right)-c_{i}\left(Y^{+}(s)\right)\right] d s \\
& +\sum_{k=1}^{n} \int_{0}^{t}\left[\sigma_{i k}\left(X^{+}(s)\right)-\sigma_{i k}\left(Y^{+}(s)\right)\right] d W^{+k}(s)
\end{aligned}
$$

almost everywhere with respect to $P^{\prime}$. Applying Theorem 5.1, we get

$$
P^{\prime}\left[X^{+}(t)>Y^{+}(t) \text { for all sufficiently small } t>0\right]=1 .
$$

But due to the continuity of $X$ and $Y$ we would expect this measure to be 0 . The contradiction arises from the assumption that $P(D)>0$, and we can therefore conclude that $P(m=+\infty)=1$. Q.E.D.

6. A uniqueness theorem for solutions of stochastic equations. Theorem 5.2 can be used to prove a uniqueness theorem for solutions of stochastic equations.

Suppose that $a: R \rightarrow R$ and $\sigma: R \rightarrow R$ are continuous functions satisfying the condition

$$
(a(x))^{2}+(\sigma(x))^{2} \leqq K_{1}\left(1+x^{2}\right), \quad x \in R,
$$

and let $X(0)$ be an $\mathscr{F}_{0}$-measurable random variable. By a theorem of A. V. Skorohod $[5$, p. 121], there is a continuous nonanticipating function $X$ such that

$$
X(t)=X(0)+\int_{0}^{t} a(X(s)) d s+\int_{0}^{t} \sigma(X(s)) d W(s), \quad t \geqq 0 .
$$

However, continuity of the functions $a(\cdot)$ and $\sigma(\cdot)$ is not sufficient to prove uniqueness of the solution $X$, i.e. that $X$ is the only continuous nonanticipating function satisfying (7). Any one of the following conditions has been shown to be sufficient to insure uniqueness of the solution $X$.

(i) $a(\cdot)$ and $\sigma(\cdot)$ satisfy Lipschitz conditions on $R$. See K. Itô [3].

(ii) $\sigma(\cdot)>0$ and $\sigma(\cdot)$ satisfies on $R$ a Hölder condition with exponent greater than $\frac{1}{2}$. See A. V. Skorohod [5].

(iii) $\sigma(\cdot)>\varepsilon>0$ for some constant $\varepsilon$, and $\sigma(\cdot)$ satisfies on $R$ a Hölder condition with positive exponent. See I. V. Girsanov [2].

As far as the strong Markov process generated by equation (7) is concerned, the condition $\sigma(\cdot)>0$ in (ii) and (iii) rules out the existence of any interesting singularities. In the following theorem, we show that if $\sigma$ has zeros, then condition (ii) is still applicable provided we assume that $a(\cdot)$ satisfies a Lipschitz condition on $R$. 
THEOREM 6.1. Suppose the function a(·) satisfies the Lipschitz condition

$$
|a(x)-a(y)| \leqq K_{2}|x-y|, \quad x, y \in R,
$$

where $K_{2} \geqq 0$ and that $\sigma(\cdot)$ satisfies the Hölder condition

$$
|\sigma(x)-\sigma(y)| \leqq K_{3}|x-y|^{\alpha}, \quad x, y \in R,
$$

where $K_{3} \geqq 0$ and $\alpha>\frac{1}{2}$.

Then for any $\mathscr{F}_{0}$-measurable random variable $X(0)$, equation (7) has only one continuous nonanticipating solution.

Proof. By a remark of A. V. Skorohod [5, p. 121] it suffices to prove the theorem under the additional assumption that $a(\cdot)$ and $\sigma(\cdot)$ are bounded, say

$$
|a(x)|+|\sigma(x)| \leqq K_{4}, \quad x \in R .
$$

Let us suppose that $X$ and $Y$ are two continuous nonanticipating solutions of equation (7). In other words,

$$
\begin{array}{ll}
X(t)=X(0)+\int_{0}^{t} a(X(s)) d s+\int_{0}^{t} \sigma(X(s)) d W(s), & t \geqq 0, \\
Y(t)=X(0)+\int_{0}^{t} a(Y(s)) d s+\int_{0}^{t} \sigma(Y(s)) d W(s), & t \geqq 0 .
\end{array}
$$

Let $\varepsilon>0$ be arbitrary and define functions $f$ and $g$ by

$$
f(\cdot)=a(\cdot)-\varepsilon, \quad g(\cdot)=a(\cdot)+\varepsilon .
$$

Now there exist continuous nonanticipating functions $U$ and $V$ satisfying the equations

$$
\begin{array}{ll}
U(t)=X(0)+\int_{0}^{t} f(U(s)) d s+\int_{0}^{t} \sigma(U(s)) d W(s), & t \geqq 0, \\
V(t)=X(0)+\int_{0}^{t} g(V(s)) d s+\int_{0}^{t} \sigma(V(s)) d W(s), & t \geqq 0,
\end{array}
$$

respectively. Because $f(\cdot)<a(\cdot)<g(\cdot)$, then

$$
\begin{array}{ll}
U(t) \leqq X(t) \leqq V(t), & t \geqq 0 \text { a.e. } \\
U(t) \leqq Y(t) \leqq V(t), & t \geqq 0 \text { a.e. }
\end{array}
$$

by Theorem 5.2. Consequently,

$$
|X(t)-Y(t)| \leqq V(t)-U(t), \quad t \geqq 0 \text { a.e. }
$$

Now

$$
V(t)-U(t)=\int_{0}^{t}[g(V(s))-f(U(s))] d s+\int_{0}^{t}[\sigma(V(s))-\sigma(U(s))] d W(s) .
$$


Because $V(t)-U(t) \geqq 0$ a. for each $t \geqq 0$ and because the expected value of the stochastic integral is zero, we can write

$$
\begin{aligned}
E|V(t)-U(t)| & =\int_{0}^{t} E[g(V(s))-f(U(s))] d s \\
& \leqq \int_{0}^{t} E|g(V(s))-f(U(s))| d s \\
& \leqq 2 \varepsilon t+\int_{0}^{t} E|a(V(s))-a(U(s))| d s \\
& \leqq 2 \varepsilon t+K_{2} \int_{0}^{t} E|V(s)-U(s)| d s .
\end{aligned}
$$

Put $h(s)=(E|V(s)-U(s)|) / 2 \varepsilon, 0 \leqq s \leqq t$, so that $h(t) \leqq t+\int_{0}^{t} h(s) d s$. Using the fact that $a(\cdot)$ is bounded, it is easy to show by iteration that $h(t) \leqq \exp \left(K_{2} t\right)-1$. Therefore

$$
E|X(t)-Y(t)| \leqq E|V(t)-U(t)| \leqq 2 \varepsilon\left(\exp \left(K_{2} t\right)-1\right) .
$$

Since $\varepsilon$ was arbitrary, we conclude that $X(t)=Y(t)$ a.e. This is true for every $t \geqq 0$, and since the functions $X$ and $Y$ are continuous,

$$
P[X(t)=Y(t) \text { for all } t \geqq 0]=1 .
$$

This completes the proof. Q.E.D.

In a sense, the criteria for uniqueness of Theorem 6.1 might be viewed as the best possible. The requirement $\alpha>\frac{1}{2}$ cannot be appreciably weakened, as is demonstrated by the Girsanov example (see H. P. McKean, Jr. [4])-if $0<\alpha<\frac{1}{2}$ the onedimensional stochastic equation $X(t)=\int_{0}^{t}|X(s)|^{\alpha} d W(s), t \geqq 0$, has infinitely many continuous nonanticipating solutions. On the other hand, it seems implausible that the Lipschitz condition on $a(\cdot)$ can be weakened. For in the extreme case where $\sigma(\cdot)=0$, the stochastic equation (7) reduces to the deterministic integral equation $X(t)=X(0)+\int_{0}^{t} a(X(s)) d s, t \geqq 0$, and here it is required in general that $a(\cdot)$ be Lipschitz.

It is also possible to prove a version of Theorem 6.1 for $n$-dimensional stochastic equations. As is suggested by the hypothesis of Theorem 5.2, we must assume that for each $i$ between 1 and $n$, the functions $\sigma_{i k}(\cdot), k=1, \ldots, n$, depend only on the $i$ th component $x_{i}$ of $x \in R^{n}$. This has the undesirable effect of reducing the $n$ dimensional equation to a series of $n$ one-dimensional equations. For this reason we have preferred to prove Theorem 6.1 in the one-dimensional case.

\section{BIBLIOGRAPHY}

1. D. A. Dawson, Equivalence of Markov processes, Trans. Amer. Math. Soc. 131 (1968), 1-31. MR 37 \#5937.

2. I. V. Girsanov, On Ito's stochastic integral equation, Dokl. Akad. Nauk SSSR 138 (1961), 18-21 = Soviet Math. Dokl. 2 (1961), 506-509. MR 22 \#10010. 
3. K. Itô, Lectures on stochastic processes (notes by K. M. Rao), Tata Institute of Fundamental Research, Bombay, 1961.

4. H. P. McKean, Jr., Stochastic integrals, Probability and Math. Statist., no. 5, Academic Press, New York, 1969. MR 40 \#947.

5. A. V. Skorohod, Studies in the theory of random processes, Izdat. Kiev. Univ., Kiev, 1961; English transl., Addison-Wesley, Reading, Mass., 1965. MR 32 \#3082a, b.

Westfield College, University of London, London, England

Current address: Department of Mathematics, McGill University, Montreal, Canada 